\title{
Multi-Frequency Study of the SZ Effect in Cosmic Structures
}

\author{
Sergio Colafrancesco ${ }^{1}$ \\ ${ }^{1}$ University of the Witwatersrand, Johannesburg (South Africa) \\ Corresponding author: Sergio Colafrancesco. Email: sergio.colafrancesco@wits.ac.za
}

\begin{abstract}
The Sunyaev-Zel'dovich effect (SZE) is a relevant probe for cosmology and astrophysics. A multi-frequency approach to study the SZE in cosmic structures turns out to be crucial in the use of this probe for astrophysics and cosmology. Astrophysical and cosmological applications to galaxy clusters, galaxies, radiogalaxies and large-scale structures are discussed. Future directions for the study of the SZE and its polarization are finally outlined.
\end{abstract}

Keywords: cosmology - CMB - dark matter - dark energy - cosmic magnetism - cosmic structures: galaxy clusters, galaxies, radio galaxies.

\section{Introduction}

Galaxy clusters, the largest gravitationally bound structures in the universe, are the representative systems of the distribution of LSS in the universe. The description of these cosmic structures is continuously enriching of physical details regarding their matter and field content.

Dark Matter (DM) is the dominant form of matter that creates the potential wells of cosmic structures, from those on the largest scales down to galactic and sub-galactic scales. If we consistently take into account the fundamental nature of DM particles, we are inevitably bound to consider the effects of their annihilation or decay on the structure and evolution of the DM clumps [13].

Baryonic material collected in the DM potential wells of LSS is likely heated and shocked by large-scale shock waves found in cosmological simulations (see, e.g, [53]) which produce a complex distribution of Mach numbers. The presence of shock waves with relatively high Mach numbers naively suggest that Fermi-like acceleration might take place in LSS thereby accelerating cosmic rays $(\mathrm{CRs})$ that can be efficiently confined mainly in galaxy clusters [8], while they can diffuse out of galactic and sub-galactic structures $[8,9]$.

Radio observations and magneto-hydrodinamic (MHD) simulations also suggest that magnetic fields are associated to the distribution of baryonic material collected in LSS potential wells. The seed magnetic field is likely amplified and made turbulent by the coupling of gravitational collapse and MHD processes during the formation of galaxy clusters. Evidence for wide-scale and turbulent intra-cluster magnetic field is indicated by the diffuse radio synchrotron emission found in many clusters (with typical values $B \sim 0.1-2 \mu \mathrm{G}$ ), by radio relics in dynamically active clusters (with typical values $B \sim 0.2-5 \mu \mathrm{G}$ ) and by Faraday rotation measurements of background and embedded polarized radio sources (with typical values $B \sim 1-50 \mu \mathrm{G}$ [27].

Very massive DM clumps that collapse at high redshifts $(z \sim 6-7)$ often contain the most massive Black Holes (BHs) at their centers [35]. The AGN descendants of these ancient supermassive $\mathrm{BHs}$ (SMBHs) are found to be part of the massive galaxies located at the centers of the most massive galaxy clusters we observe at the present epoch, like, e.g., CenA, M87/Virgo, Perseus, A262, A4059, with their radio lobes penetrating the ICM for tens or hundreds of kpcs. It is often observed that the radio jets/lobes end up in approximately spherical bubbles of relativistic plasma (likely containing relativistic or mildly-relativistic plasmas) that appear as cavities in the X-ray images of galaxy clusters [3] with dimensions ranging from a few kpcs (as in Perseus) to $\sim 100 \mathrm{kpc}$. The combination of high-resolution radio and X-ray images indicates that the relativistic plasma found inside the X-ray cavities is connected with the jet/lobe structure of the central AGN and with the history of the ejection events and mechanisms from the central AGN.

The cluster cores which host such non-thermal phenomena (BHs, cavities, magnetic fields) are found to be systematically cooler than the outer regions of the clusters with the inner temperature setting at a value $\sim \frac{1}{3}-\frac{1}{2}$ of the outer temperature, usually consistent with the virial expectation. It seems that a heating agent of nongravitational and non-thermal nature - with a heating rate that is able to accomodate itself to the cooling rate 
of the intra-cluster plasma - is present in the cluster's cool core so as to maintain it in a quasi-stationary, warm configuration [16].

The previous evidence indicates that galaxy clusters are the largest storage rooms for cosmic material (galaxies, DM, hot thermal baryonic plasma, non-thermal and relativistic plasma, BHs, magnetic fields, CRs). In this sense they can be considered as the largest multidisciplinary laboratories in the universe where one can efficiently study some of the most interesting aspects of the astrophysics of LSS: the nature of DM, the origin and distribution of CRs, the impact of magnetic fields on LSS, the impact of BHs on LSS, the interplay between thermal and non-thermal phenomena in LSS.

Two ways can be identified to proceed in this study: i) a multi-technique, single-purpose approach that require to integrate the analysis of various observations performed in various frequency bands; ii) a singletechnique, multipurpose approach that is able to provide detailed physical information based on a single observational technique. We will discuss, in the following, some of the results obtainable through the last approach by using the physical information contained into the SZE.

\section{The Physics of the SZ Effect}

The SZE is produced by the inverse Compton scattering (ICS) of CMB photons off the electrons confined in the atmospheres of cosmic structures. Manifestations of the SZE include: i) spectral distortions of the CMB due to up-scattering of CMB photons induced by high-E electrons (thermal, non-thermal and relativistic SZE); ii) spectral distortion of the CMB due to a bulk motion of the electronic plasma w.r.t. the Hubble flow (kinematic SZE); iii) polarization of the CMB due to dynamical and plasma effects (SZE polarization).

The SZ effect. The spectral distortion of the CMB spectrum observable in the direction of a galaxy cluster writes $[56,2,6]$ as

$$
\Delta I(x)=2 \frac{\left(k T_{C M B}\right)^{3}}{(h c)^{2}} y g(x),
$$

where $\Delta I(x)=I(x)-I_{0}(x), I(x)$ is the up-scattered CMB spectrum in the direction of the cluster and $I_{0}(x)$ is the unscattered CMB spectrum in the direction of a sky area contiguous to the cluster. Here $x \equiv h \nu / k T_{C M B}, h$ is the Planck constant, $k$ is the Boltzmann constant, $T_{C M B}=2.726 \mathrm{~K}$ is the CMB temperature today and $\nu$ is the observing frequency. The Comptonization parameter $y$ is

$$
y=\frac{\sigma_{T}}{m_{\mathrm{e}} c^{2}} \int P_{\mathrm{e}} d \ell
$$

in terms of the pressure $P_{\mathrm{e}}$ contributed by the electronic population. Here $\sigma_{T}$ is the Thomson cross section, $m_{e}$ the electron mass, and $c$ the speed of light. The spectral function $g(x)$ of the SZE is

$$
g(x)=\frac{m_{\mathrm{e}} c^{2}}{\left\langle\varepsilon_{\mathrm{e}}\right\rangle}\left\{\frac{1}{\tau_{e}}\left[\int_{-\infty}^{+\infty} i_{0}\left(x e^{-s}\right) P(s) d s-i_{0}(x)\right]\right\}
$$

[6] in terms of the photon redistribution function $P(s)$ and of

$$
i_{0}(x)=I_{0}(x) /\left[2\left(k T_{C M B}\right)^{3} /(h c)^{2}\right]=x^{3} /\left(e^{x}-1\right) .
$$

The quantity

$$
\left\langle\varepsilon_{\mathrm{e}}\right\rangle \equiv \frac{\sigma_{\mathrm{T}}}{\tau_{e}} \int P_{e} d \ell=\int_{0}^{\infty} d p f_{\mathrm{e}}(p) \frac{1}{3} p v(p) m_{\mathrm{e}} c,
$$

where $f_{e}(p)$ is the normalized electron momentum distribution function, is the average energy of the electron plasma [6]. The optical depth along the line of sight $\ell$ of the electron population with number density $n_{e}$ is

$$
\tau_{\mathrm{e}}=\sigma_{T} \int d \ell n_{\mathrm{e}} .
$$

The photon redistribution function $P(s)$, with $s=$ $\ln \left(\nu^{\prime} / \nu\right)$ in terms of the CMB photon frequency increase factor $\nu^{\prime} / \nu$, can be calculated by repeated convolution of the single-scattering redistribution function, $P_{1}(s)=\int d p f_{\mathrm{e}}(p) P_{\mathrm{s}}(s ; p)$, where $P_{s}(s ; p)$ depends on the physics of inverse Compton scattering. The previous description is relativistically covariant and general enough to be applied to both thermal and nonthermal plasma, as well as to a combination of the two (see $[6,17,38]$ for details).

Kinematic SZ effect. The velocity (or kinematic) SZE (hereafter kSZE) arises if the plasma causing the thermal, or non-thermal, SZE is moving relative to the Hubble flow. In the reference frame of the scattering particle the CMB radiation appears anisotropic, and the effect of the ICS is to re-isotropize the radiation slightly. Back in the rest frame of the observer the radiation field is no longer isotropic, but shows a structure towards the scattering atmosphere with amplitude $\propto \tau_{e} V_{t} / c$, where $V_{t}$ is the component of the peculiar velocity of the scattering atmosphere along the line of sight $[55,51]$. The brightness change of the $\mathrm{CMB}$ due to the kSZE is given by

$$
\frac{\Delta I}{I}=-\tau_{e} \beta_{t} \frac{x e^{x}}{e^{x}-1}
$$

with $\beta_{t} \equiv \frac{V_{t}}{c}[55,40]$. A general relativistic description of the kSZE has been given in the framework of the general Boltzmann equation [29] and in the relativistic covariant formalism [38].

SZ effect polarization. The ICS process yields naturally a polarized upscattered radiation field. The polarization $\Pi$ of the SZE arises from various dynamical 
and plasma effects $[54,22,32,1]$ : galaxy clusters transverse motion $\left(\Pi_{k} \propto \beta_{t}^{2} \tau\right.$ in the Rayleigh-Jeans, RJ, regime), transverse motions of plasma within the cluster $\left(\Pi_{v} \propto \beta_{t} \tau^{2}\right.$ in the RJ regime) and multiple scattering between electrons and CMB photons within the cluster $\left(\Pi_{t h} \propto \Theta \tau^{2}\right.$ in the RJ regime for the thermal SZE with $\Theta=k T_{e} / m_{e} c^{2}$ ). A general, covariant, relativistic derivation of the SZE polarization for thermal, non-thermal and relativistic plasma can be derived [22] and generalizes the non-relativistic derivation [54] in a way similar to the general derivation of the SZE [6] previously discussed.

\section{Astrophysical and Cosmological Impact}

Studying the SZE in the atmospheres of various cosmic provides many insights on their energetics, pressure and dynamical structure. The combination of SZE with other emission mechanisms related to the same particle distribution (i.e., synchrotron, high-E ICS and bremsstrahlung emission) provides further information on the radiation, matter and magnetic fields that are co-spatial with the electrons producing the SZE. These properties of the SZE concern various cosmic structures, from galaxy clusters to radiogalaxy lobes, from galaxy halos to supercluster.

The redshift-independent nature of the SZE allows to use it as a powerful cosmological probe yielding constraints on the evolution of cluster abundance, cosmological parameters, the Dark Energy equation of state, the homogeneity of the universe, the properties of cosmological magnetic fields [14]. Observations aimed at these challenges must however exploit the whole physical information contained in the spectral and spatial features of the SZE.

\subsection{Simple SZE astrophysics}

The SZE has been searched in galaxy clusters since it was originally proposed $[55,56]$ using various techniques (see [14] for a recent review). Ground-based SZE experiments (e.g., SPT, ACT, APEX, AMI, GBT, among others) provided excellent results in terms of imaging and blind search surveys with their low frequency, multiple-band observations, but they do not have neither true spectroscopic capabilities nor a wide frequency band, and they are not sensitive to the high- $\nu$ range ( $\gtrsim 400 \mathrm{GHz}$ ) of the SZE signals, which is crucial to exploit the astrophysical information contained in the SZE (see Fig. 1, [14]). Ground-based instruments widely improved the source statistics (crucial to obtain cosmological information using the SZE) and the angular resolution of SZE images (crucial to disentangle the extended SZE signal from point-source contamination), but add little to the physical specification of the detected SZE sources, and therefore they need X-ray and optical follow-up to fully characterize the physical parameters derived from SZE observations.

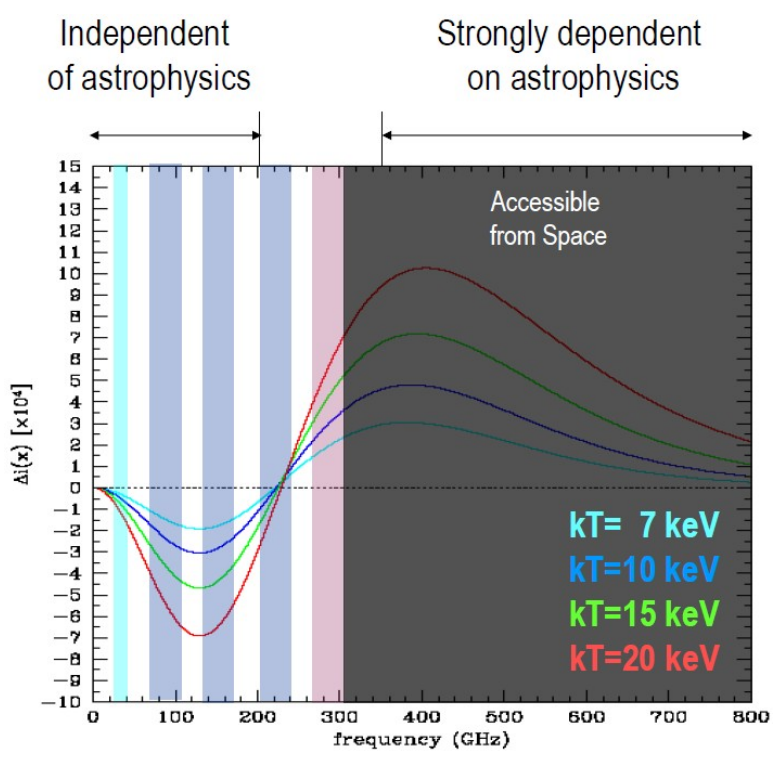

Figure 1: Thermal SZE spectrum of galaxy clusters with different plasma temperatures (as indicated) and the same value of the optical depth. The low- $\nu$ part $(\nu \lesssim 220 \mathrm{GHz})$ of the spectrum depends mostly on the total Compton parameter $y \propto \int d \ell P_{t o t}$ with no strong spectral dependence on the temperature. The high- $\nu$ part of the spectrum (at $\gtrsim 300 \mathrm{GHz}$ ) shows a strong spectral dependence from the plasma temperature [17]. Typical frequency bands where the SZE is observed from the ground are shown as blue-cyan bands, while the region accessible from space observations is shown by the gray shaded area.

\subsection{The road to astrophysics}

The Planck satellite allowed for the first time to access a wide frequency range in the study of the SZE. Planck early SZE science observations yielded 189 SZE sources with $\mathrm{S} / \mathrm{N}>6$ which provides the first SZE measure for $\sim 80 \%$ of the known galaxy clusters, and 20 additional new clusters (see Arnaud at this Meeting). This is the largest sample so far of SZE detected clusters. Planck detected SZE clusters are followed-up with a multi-frequency observation program in the X-rays, SZE, optical bands to obtain confirmation, redshift estimation and estimates of the global physical parameters. These results show that the SZE selection is a very powerful method for the detection of new distant and very massive clusters. Planck also unveiled a population of dynamically perturbed clusters at $z \gtrsim 0.3$, 
possibly underrepresented in X-ray surveys. The information collected so far strengthen our overall view of the ICM properties and mass content in galaxy clusters. Most of these results are discussed in the early and intermediate papers [41, 42, 43, 45, 46, 47] and more analyses are still coming. The use of Planck SZE results also allowed to extend the sample of radio halo clusters with combined radio, SZE and X-ray data [23]. The correlation $Y_{S Z}-L_{X}$ probes the existence of a substantial amount of non-thermal pressure in clusters that also requires a correlation $X \propto L_{X}^{-0.96}$ between the nonthermal to thermal pressure ratio $X=P_{n o n-t h} / P_{t h}$ and the cluster X-ray luminosity $L_{X}$ [23]. The nature of the non-thermal electron population could be only probed with additional high- $\nu$ spectroscopic studies.

The Herschel satellite (co-eval with Planck) has been able to observe the SZE in a few pointed clusters with the Spire instrument equipped with an FTS spectrometer working in the frequency range $\sim 600-1200$ GHz. The possibility to have sensitive spectroscopic measurements in these high-frequency bands opens the way to the deep astrophysical exploitation of the SZE. As an example, the additional data points on the SZE spectrum of the Bullet cluster observed with HerschelSpire [60] allowed to establish a number of properties for the thermal and non-thermal plasma superposition in the atmosphere of this strong merging cluster (see, e.g. $[18,48,49]$. A consequence of such superposition is that cluster temperature distribution has a high value of of the temperature standard deviation $\sigma_{T}$, as in the case of the Bullet cluster where it is found $\sigma_{T}=10.6 \pm 3.8$ $\mathrm{keV}$ [50]. This result shows that the temperature distribution in the Bullet Cluster is strongly inhomogeneous along the line of sight and provides a new method for studying galaxy clusters in depth. These studies have been possible because the access to the very high- $\nu$ part of the SZE spectrum contains detailed information on the relativistic effect on the single thermal plasma and on the presence of additional plasmas of either thermal or non-thermal nature (see discussion in $[18,21]$.

Planck and Herschel observations of the SZE opened a rich field of investigation that will fully blossom in the next years with the full exploitation of spatiallyresolved spectroscopic SZE observations. In the following we discuss some of the astrophysical and cosmological studies that will be possible with a spectropolarimetric study of the SZE.

\section{Astrophysical Impact}

Studying the SZE in various cosmic atmospheres provides many insights on their energetics, pressure and dynamical structure. The combination of SZE with other emission mechanisms related to the same particle distribution (i.e., synchrotron, high-E ICS emission, bremsstrahlung emission) provides further information on the radiation, matter and magnetic fields that are co-spatial with the electrons producing the SZE in various cosmic structures.

\subsubsection{Galaxy Clusters}

Precise observations of the SZE at $\mathrm{mm}$ and sub-mm wavelengths are crucial for unveiling the detailed structure of cluster atmospheres, their temperature distribution, and the possible presence of suprathermal and/or nonthermal plasma because the high-frequency part (i.e. at $\nu \gtrsim 350 \mathrm{GHz}$ or $x \gtrsim 6$ ) of the SZE spectrum is more sensitive to the relativistic effects of the electron momentum distribution $[6,10,17]$. This is even more so for galaxy clusters with a complex plasma distribution as found for powerful merging clusters, like the exemplary case offered by the Bullet cluster (1ES0657-56) [18]. Powerful merging events in galaxy clusters can, in fact, produce an additional high-T plasma distribution (if the electron acceleration time scale at the merging shocks is longer than their equilibration time scale [57]), or an additional nonthermal population (produced either in a merging process with a very short acceleration time scale or by secondary electrons produced by p-p collisions, after the high-E protons have been accelerated by the merging and accumulate in the cluster region on long time scales [58]). The quasi-stationary case provided by the competition between particle thermalization and stochastic acceleration and momentum diffusion [26] can develop a subrelativistic electron distribution tail and can produce suprathermal regions in the cluster atmosphere.

A quantitative estimate of the temperature inhomogeneity (stratification) along the line of sight is possible using SZE data only providing a measure the temperature standard deviation of the cluster plasma along the line of sight. We found that the Bullet cluster has a temperature standard deviation of $10.6 \pm 3.8 \mathrm{keV}$ [50]. This result (obtained for the first time with SZE measurements) shows that the temperature distribution in the Bullet cluster is strongly inhomogeneous and provides a new method for studying galaxy clusters in depth. Study of the multifrequency (from $\sim 30$ to $\sim 850 \mathrm{GHz}$ ) SZE signal observed in the Bullet cluster shows, in fact, the presence of a thermal plasma at $\sim 13.9 \mathrm{keV}$ coexisting with a second plasma component, either at higher temperature $(\approx 25 \mathrm{keV})$ or, more preferably, of a nonthermal origin [18] (see Fig.2). Additional observations of the Bullet cluster at $\nu \sim 400 \mathrm{GHz}$ with a precision $\lesssim 1 \%$ of the expected signal will be able to further distinguish between the two cases of non-thermal powerlaw or suprathermal tail [18].

SZE observations over a wide frequency range, and especially with high sensitivity in the high- $\nu$ range, can 
also add relevant information on the electron distribution function (DF) in the ICM, a subject that - even though relevant for a proper analysis of the SZE - has not been addressed in details so far. The relativistic kinetic theory, on which the DF derivation is based, is still a subject of numerous debates (see discussion in [48]). SZE observations can separate the SZE spectrum caused by a departure from the diffusive approximation based on the Kompaneets approach [31] from those which are due to using a relativistic correct DF instead of a Maxwell-Boltzman DF (see Fig.3) and therefore set constraints to the actual electron DF [48]. This analysis is best fulfilled in hot massive clusters because the SZE intensity change due to using a relativistic correct DF instead of a Maxwell-Boltzman DF are much larger in hot clusters due to the fact that relativistic SZE corrections scale as $\propto T^{5 / 2}$. A method used to derive the $\mathrm{DF}$ of electrons using SZE multi-frequency observations of massive hot clusters [48] makes use of Fourier analysis representation of the approximate electron DF whose parameters are best fitted using observations in the (optimal) frequency channels at 375, 600, 700, $857 \mathrm{GHz}$.

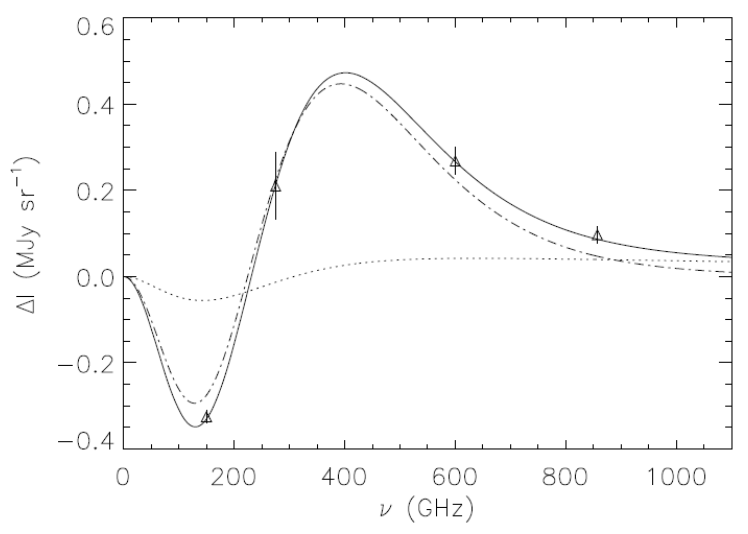

Figure 2: The SZE spectrum at the Bullet cluster center modeled with a thermal plus nonthermal plasma: thermal plasma with $k T=13.9 \mathrm{keV}$ and $\tau=1.1 \times 10^{-2}$ (dot-dashed); nonthermal plasma with $p_{1}=1, s=2.7$ and $\tau=2.3 \times 10^{-4}$ (dotted); total SZE produced by the sum of the two plasmas (solid).

A morphological analysis of the SZE observed at various frequencies adds relevant information to assess the pressure and energy density structure of cluster atmospheres. Morphological SZE differences are particularly evident for clusters undergoing violent mergers that create large inhomogeneities of the electron DF. SZE intensity maps of merging clusters obtained from hydrodynamical simulations show that the morphology of the SZE intensity maps observable with LABOCA (at $345 \mathrm{GHz}$ ) and Herschel-Spire (at $857 \mathrm{GHz}$ ) are rather different [49] (see Fig.4). For a Bullet-like cluster, the SZE intensity map at $857 \mathrm{GHz}$ has a spatial feature caused by the presence of the cold Bullet-like substructure seen also in the X-ray surface brightness map. However, this cold substructure is not present in the SZE intensity map at $345 \mathrm{GHz}$. This is a consequence of the relativistic effects of the SZE and shows that observations of the SZE intensity maps at very high frequencies can reveal complex pressure substructures within the atmospheres of massive galaxy clusters. This result shows that the analysis of the SZE signal at $857 \mathrm{GHz}$, correlated with lower- $\nu$ observations offers a promising method for unveiling high- $T$ regions in massive merging clusters using available experiments like, e.g LABOCA and Herschel-Spire.

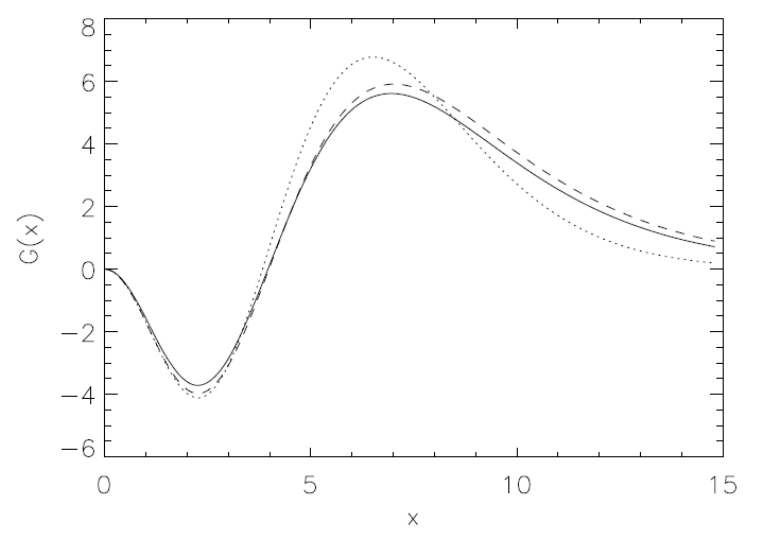

Figure 3: The SZE intensity spectra for a massive cluster with a temperature of $k T_{e}=15.3 \mathrm{keV}$ for Juttner (solid) and Maxwell-Boltzman (dashed) DFs. The non-relativistic SZE spectrum (solid) is also shown for comparison. Figure from [48]

In more relaxed clusters spectroscopic measurements of the SZE over a wide frequency band allow to derive precise information on the temperature distribution and on the cool-core nature independently of X-ray priors [17] and hence reconstruct the full set of cluster physical parameters [25].

Spectro-polarimetry measurement of the SZE are able to add further information on the transverse plasma motions within the cluster and on the pressure substructure of the plasma. SZE polarization signals in galaxy clusters are quite low and typically below mJy (or $\approx \mu \mathrm{K})$ level even for high- $T$ clusters [14] but it is interesting to note that the SZE polarization in cluster has quite different spectra w.r.t. the intensity SZE spectrum, a property that requires to use spectropolarimetry to fully disentangle the physical information contained in the SZE. Combining intensity and polarization observations of the SZE can uncover unique 
details of the 3d (projected and along the line of sight) velocity structure of the ICM, of its $3 \mathrm{~d}$ pressure structure and of the influence of a structured magnetic field in the stratification of the ICM, and therefore provides a full tomography of cluster atmospheres. Analogously, the combination of the intensity and polarization observations of the kinematic SZE (and its frequency dependence) can yield crucial information on the $3 \mathrm{~d}$ distribution of the cosmological velocity field traced by galaxy clusters. Specifically, the ratio $\Delta I_{t h} / \Pi_{t h}$ yields direct information on the plasma optical depth $\tau$, and the ratio $\Delta I_{t h} / \Pi_{v}$ on the combination $\tau \cdot \beta_{t}$, thus allowing to use intensity and polarization SZE measurements to fully disentangle the pressure and velocity structure of the cluster atmospheres. SZE polarization measurements are quite difficult to obtain with present-day experiments and they are also at the limit of next generation experiments. However, stacking analysis of even small samples (order of $\sim 20$ ) of hot and dense galaxy clusters would allow to determine statistically the polarization signals of the thermal SZE for clusters with $k T>10 \mathrm{keV}$ and $\tau>0.03$ in the optimal frequency range $\approx 90-250 \mathrm{GHz}$.
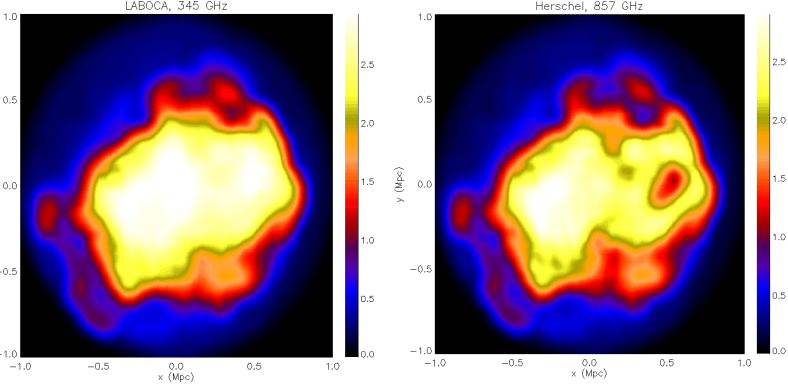

Figure 4: From top to bottom. The SZE signal to noise ratio map for the cluster 1E0657-558 at $345 \mathrm{GHz}$ smoothed to the resolution of LABOCA and at $857 \mathrm{GHz}$ smoothed to the resolution of Herschel-Spire. Figures from [49].

\subsubsection{Cluster cavities}

The atmospheres of galaxy clusters often show the presence of bubbles filled with high-E particles and magnetic field that are sites of bright radio emission and produce cavities in their X-ray emission distribution. Cavities with diameters ranging from a few to a few hundreds of kpc have been observed by Chandra in the X-ray emission maps of several galaxy clusters and groups [3, 34]. While the properties of these cavities and of the relativistic plasma they contain is usually studied by combining $\mathrm{X}$-ray and radio observations, an alternative and efficient strategy is to study the consequences of the SZE produced by the high-energy electrons filling the cavities [7,39] whose amplitude, spectral and spatial features depend on the overall pressure and energetics of the relativistic plasma in the cavities. As an example, the overall SZE observable along the line of sight (LOS) through a cluster containing cavities (see Fig.5 for the case of the cluster MS0735.6+7421) is the combination of the non-thermal SZE produced by the cavity and of the thermal SZE produced by the surrounding ICM. Due to the different $\nu$-dependence of the thermal and non-thermal SZE, the non-thermal SZE from a cluster cavity shows up uncontaminated at frequencies $\nu \approx 220$ GHz: at this frequency, in fact, the overall SZE from the cluster reveals only the ICS of the electrons residing in the cavities without the presence of the intense thermal SZE dominating at lower and higher frequencies. The cavity's SZE becomes dominant again at very high- $\nu$ ( $x \gtrsim 14$ or $\nu \gtrsim 800 \mathrm{GHz})$ where the nonthermal electrons dominate the overall ICS emission (see Fig.5). The cavity's SZE is more spatially concentrated than the overall cluster SZE because it is only emerging from the cavity regions: this fact allows to study the overall energetics and pressure structure of the cavity's high-E particle population and the B-field structure in combination with X-rays and radio images. The observation of the crossover of the non-thermal SZE from the cavities (which depends on the value of $E_{\min }\left(p_{1}\right)$ or, equivalently, on the value $P_{\text {cavity }}$ ) provides a way to determine the total pressure and hence the nature of the electron population within the cavity [7], an evidence which adds crucial, complementary information to the X-ray and radio analysis.

If cluster cavities contain a high- $\mathrm{T}\left(\sim 10^{9}-10^{10} \mathrm{~K}\right)$ plasma, the SZE flux from cocoons in the central part of a distant elliptical and a nearby galaxy cluster are of the same order. For a high-T plasma, the cocoon's SZE spectrum is rather flat at high- $\nu$ resembling the shape of the non-thermal SZE from cavities. In this high-T plasma model, however, no strong radio emission at $\nu \gtrsim 1 \mathrm{GHz}$ (as instead observed) is expected from the cocoon, unless the cocoon's B-field is very high $B \gtrsim 10^{3} \mu \mathrm{G}$.

\subsubsection{Radiogalaxy lobes}

Studies of (giant) radio-galaxy (RG) lobes (see, e.g., $[28,30,24,4])$ have shown that these extended structures contain relativistic electrons that are currently available to produce both low- $\nu$ synchrotron radio emission and ICS of the CMB (as well as other radiation background) photons. As a consequence, an SZE from the lobes of RGs is inevitably expected [11]. Such nonthermal, relativistic SZE has a specific spectral shape that depends on the shape and energy extent of the spectrum of the electrons residing in RG lobes. The 
SZE emission from RG lobes is expected to be co-spatial with the relative ICS X-ray emission [11] and its spectral properties are related to those of the relative ICS $\mathrm{X}$-ray emission. In fact, the spectral slope of the ICS X-ray emission $\alpha_{X}=(\alpha-1) / 2\left(\right.$ where $\left.F_{I C S} \sim E^{-\alpha_{X}}\right)$ can be used to set the electron energy spectral slope $\alpha$ (where $N_{e} \sim E^{-\alpha}$ ) necessary to compute the SZE spectrum, and to check its consistency with the synchrotron radio spectral index $\alpha_{r}=(\alpha-1) / 2$ (where $\left.F_{\text {Synch. }} \sim E^{-\alpha_{r}}\right)$, that is expected to have the same value $[11,19]$.
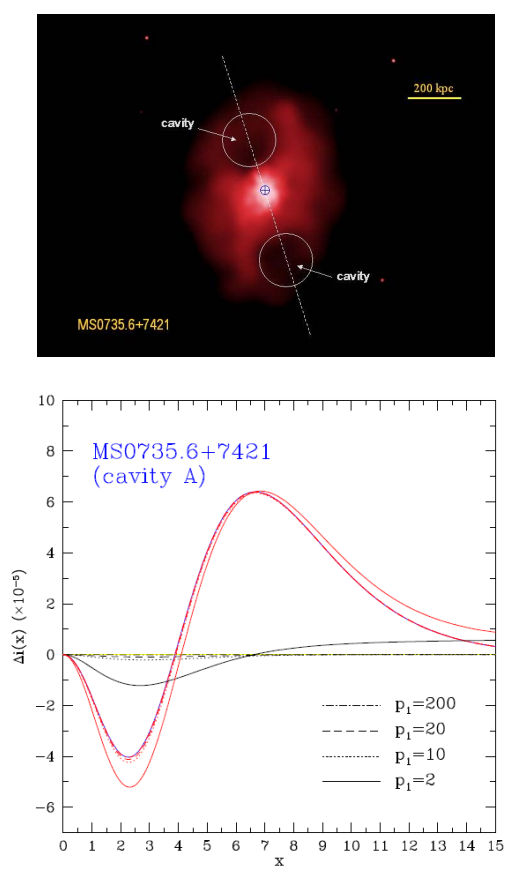

Figure 5: Top. The geometry of the cavities in the cluster MS0735.6+7421. Bottom. The SZE spectrum has been computed at a projected radius of $\approx 125 \mathrm{kpc}$ from the cluster center where the LOS passes through the center of northern cavity. The thermal SZE (blue), the non-thermal SZE from the cavity (black) and the total SZE (red) are shown. The non-thermal SZE is normalized to the cavity pressure $P=6 \cdot 10^{-11} \mathrm{erg} \mathrm{cm}^{-3}$, and is shown for various values of $p_{1}$. Figure from [7]

The SZE in RG lobes has not been detected yet: only loose upper limits have been so far derived on the SZE from these sources (see $[2,59]$ ). A detection of the SZE from RG lobes can provide a determination of the total energy density and pressure of the electron population in the lobes [11] allowing to determine the value of $E_{\min }$ once the slope of the electron spectrum is determined from radio and/or X-ray observations. SZE measurements provide a much more accurate estimates of the electron pressure/energy density than with other technique like ICS X-ray emission or synchrotron radio emission, since the former can only provide an estimate of the electron energetics in the high-energy part of the electron spectrum, and the latter is sensitive to the degenerate combination of the electron spectrum and of the magnetic field in the radio lobes. The combination of SZE observations (that depend on the electron distribution and on the known CMB radiation field) and the radio observations (which depend on the combination of the electron distribution and of the magnetic field distribution) provides an unbiased estimate of the overall B-field in the lobe by using the ratio $F_{\text {radio }} / F_{S Z E} \approx \mathcal{E}_{B} / \mathcal{E}_{C M B}$, that is more reliable than that obtained from the combination of ICS X-ray (or gamma-ray) and radio emission [19]. The spatially resolved study of the SZE and synchrotron emission in RG lobes also provide indication on the radial behaviour of both the leptonic pressure and of the magnetic field from the inner parts to the boundaries of the lobes. Study of the pressure evolution in RG lobes can provide crucial indications on the transition from radio lobe environments to the atmospheres of giant cavities observed in cluster atmospheres, which seem naturally related to the penetration of RG jets/lobes into the ICM (see Fig.5).

A substantial SZE polarization is also expected in RG lobes due to both coherent transverse motions of the plasma along the jet/lobe direction and to the electron pressure substructures induced by e.g. plasma inhomogeneities and magnetic field turbulence. The transverse velocity-induced polarization is $\Pi_{v} \propto \tau_{r e l}\left(\beta_{t} \tau_{r e l}\right)$, and the multiple scattering induced polarization is $\Pi_{\tau} \propto$ $\tau_{r e l} P_{r e l}$ where $P_{r e l}$ is the pressure of the relativistic electron distribution. Observations of the SZE and its polarization in RG lobes can yield, therefore, direct information on electrons $\tau_{r e l}$ and $\beta_{t}$ in the RG lobe.

\subsubsection{Galaxies}

Hot gas trapped in a DM halo can produce a SZE. A typical galaxy halo might hence show an integrated thermal SZE at the level of $\lesssim 0.5 \mathrm{mJy} \operatorname{arcmin}^{-2}$ from a plasma with $T \sim 10^{6} \mathrm{~K}$ and density $n_{e} \sim 10^{-3} \mathrm{~cm}^{-3}$ extended for $\sim 50 \mathrm{kpc}$ in the inter-stellar medium. A measurement of galaxy halo SZE would provide direct information on the mass, spatial distribution and thermodynamic state of the plasma in a low-mass galactic halo, and could place important constraints on current models of galaxy formation. Detecting such an extended, low-amplitude signal will be challenging, but possible with sensitive all-sky SZE maps.

An SZE is also expected from galaxy outflows swept by galaxy (hyper-)winds. A thermal SZE is expected to arise from the shocked bubble plasma in a galaxy wind described by a simple, spherical blast wave model [52]. 
However, such simple recipe for the SZE from galaxy winds is to be modified by the presence of cosmic rays and magnetic field in the expanding wind leading on one side to a more complex SZE spectrum, and on the other side to an amplification of the overall SZE at high frequencies, thus increasing the detection probability. SZE observations from galaxy winds will be possible with high-sensitivity and high-resolution telescopes like ALMA and SKA.
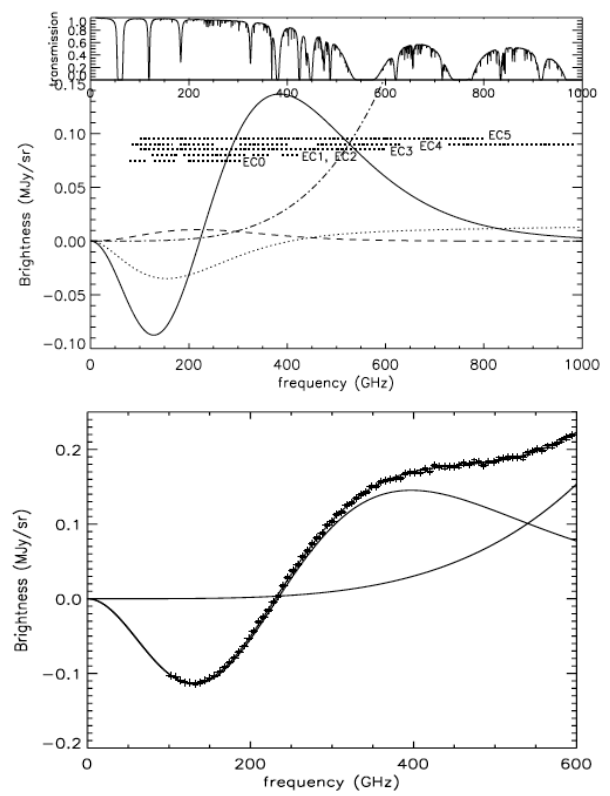

Figure 6: Top. Thermal SZE spectrum (continuous line in bottom panel), compared to the atmospheric transmission of a dry, cold, atmosphere (top panel, $\mathrm{PWV}=0.5 \mathrm{~mm}$ ), and to spectra of the nonthermal SZE (dotted line), of CMB anisotropy and kinematic SZE (dashed line), and of dust anisotropy (dotdashed line). The parameters of the different spectra are for $\tau_{t h}=5 \times 10^{-3}, T_{e}=8.5 \mathrm{keV}, \Delta T_{C M B}=$ $22 \mu \mathrm{K}, \tau_{\text {non-th }}=10^{-4}, \alpha=-2.7, p_{1}=1.4 \mathrm{MeV} / \mathrm{c}$, $\Delta I_{d}(150 G H z)=600 \mathrm{Jy}$. The frequency coverage of different experiments [25] is shown as dotted horizontal lines, labelled with the experimental configuration number. Bottom. Simulated data sets for the spectroscopic configurations EC5 (differential cold FTS on a L2-orbit satellite, with cold telescope).

\section{Cosmology and Fundamental Physics}

The redshift-independent nature of the SZE allow to use this effect as a powerful cosmological probe [14] by using both the redshift evolution of cluster abundance and direct probes of cosmological parameters. The recent Planck results however indicate a tension at $\sim 3 \sigma$ level on the value of $\sigma_{8}$ vs. $\Omega_{m}$ comparing
SZE counts and CMB results that might be mitigated by other cosmological effects like, e.g., a more complex cluster bias dependence, the existence of massive neutrinos with $\sum m_{\nu} \sim 0.2 \mathrm{eV}$ (see Rubino-Martin in these proceedings) or by addressing more in details the complex astrophysics issues still pertaining cluster formation and evolution. This last possibility requires to have new high-quality SZE cluster samples studied with full spectra-polarimetric capabilities and in the high- $\nu$ range of the SZE spectra where the astrophysical effects are more evident. Fig. 6 shows the frequency coverage requirements for a full spectroscopic SZE mission and the outcomes of a simulated thermal SZE spectrum from a $k T_{e}=8.5 \mathrm{keV}$ cluster [25]. The sensitivity and spectro-polarimetry power of such an experimental configuration (EC5) will allow to address fundamental questions in cosmology and fundamental astrophysics with the SZE, in addition to standard cosmological probes [14].

A wide discussion on the cosmological relevance of the SZE for probing the concordance cosmological scenario, the search for the nature of DM, cosmological magnetic fields and cosmological velocity fields can be found in [14]. Here we discuss the additional application of the spectro-polarimetric observations of the SZE to measure the CMB multiplies and test the assumption of the homogeneity of the Universe. High-sensitivity observations of the (thermal and kinematic) SZE and its polarization can be used to test the homogeneity of our universe through probes of the Copernican Principle (CP) [33, 23]. In fact, large variations of the thermal SZE induced by CMB photons that have temperature significantly different from the blackbody temperature we observe directly, and that arrive at galaxy cluster from points inside our light cone, could indicate a violation of the $\mathrm{CP}$ and homogeneity. Analogously, large variations of the $\mathrm{CMB}$ dipole measured by the cluster kSZE could also indicate a violation of the $\mathrm{CP}$ and homogeneity. The SZE polarization contains more refined information on the CMB temperature and thus it can also provide a powerful probe of $\mathrm{CP}$ and homogeneity. Thus, observations of large SZE and of its polarization w.r.t. to the expectations of the SZE produced from a pure blackbody CMB spectrum might provide indications of a non-FLRW universe.

The combination of an EC5 experiment with radio observations of the low- $\nu$ part of the SZE spectrum can also be used to probe even more fundamental aspects of astrophysics, like the fundamental properties of the photon. Measurements of the SZE with sensitivity of order of $<0.1 \mathrm{Jy}$, in the range $\sim 10-50 \mathrm{GHz}$, can set very stringent constraints on the photon decay time [20]. This frequency band is also the one less affected by other sources of astrophysical contamination, and it will be best explored with the advent of the high- 
sensitivity SKA telescope. At higher frequencies (i.e., $\sim 120-180$ and $\sim 200-300 \mathrm{GHz}$ ), there are other spectral windows where the SZE method is again competitive if not advantageous in this respect. The necessary sensitivity in the high- $\nu$ range can be achieved with the next coming Millimetron space mission [14], whose EC5-like configuration is also advantageous in disentangling other astrophysical sources of contamination (e.g., sources of non-thermal SZE and/or special distortions of the SZE due to multiple-temperature regions) that could contaminate these measurements.

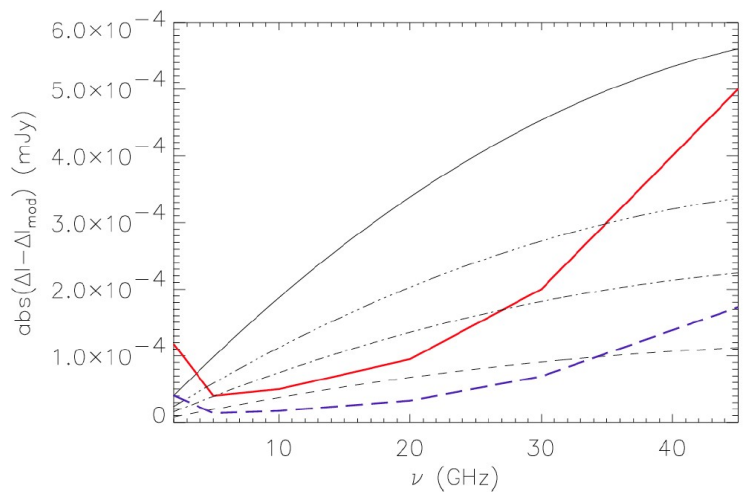

Figure 7: Difference between the standard thermal SZE calculated for A2163 in 2-45 GHz range and integrated in a $1 \operatorname{arcmin}^{2}$ area and the one modified for values $E^{*}=5 \times 10^{-9} \mathrm{eV}$ (solid line), $3 \times 10^{-9} \mathrm{eV}$ (dash - 3 dots), $2 \times 10^{-9} \mathrm{eV}$ (dot-dashed), and $1 \times 10^{-9} \mathrm{eV}$ (dashed). The sensitivity achievable with SKA in $\sim 30$ hours (red thick solid line) and in $\sim 260$ hours (blue thick long-dashed line) are shown [20]

\section{$6 \quad$ Future Directions}

The impact of SZE observation for astrophysical and cosmological application is steadily increasing since the advent of dedicated experiments and large-scale surveys in the $\mathrm{mm}$ and sub-mm frequency range. The quality and the spatial resolution of SZE images is reaching the level of arcsecs and the spectral coverage in systematically extending in the sub-mm region where highest spatial resolutions can be achieved with a wide spectral coverage able to decipher the physical details of the electron distribution in the atmosphere of cosmic structures.

New paths of theoretical investigations are underway and concern both the detailed study of the ICS mechanism and the impact of the various plasma structure and fields in cosmic structures. The possibility to perform precise measurements of the various SZE signals and to extract the relevant astrophysical information depends crucially on the capability to have spatially-resolved spectral (and polarization) observations of SZE sources over a wide $\nu$ band, from radio to sub-mm. In particular, the important condition for such study is to have a wide-band continuum spectroscopy (polarimetry) and especially a good spectral coverage and sensitivity in the high- $\nu$ band, where most of the astrophysical effects reveals more clearly. Spatially resolved spectroscopic and polarimetric observations of the SZE in the frequency range from $\sim 100$ $\mathrm{GHz}$ to $\sim 1 \mathrm{THz}$, complemented by analogous observations in the radio band $\sim 1-30 \mathrm{GHz}$, are the key to improve our understanding of the structure of cosmic atmospheres through analysis of the intensity and polarized SZE signal, and will allow to use this technique to probe the fundamental properties of the universe.

\section{Acknowledgement}

S.C. acknowledges support by the South African Research Chairs Initiative of the Department of Science and Technology and National Research Foundation and by the Square Kilometre Array (SKA).

\section{References}

[1] Baumann, D. \& Cooray, A. 2003, New Astron.Rev. 47, 839 doi:10.1016/j.newar.2003.07.013

[2] Birkinshaw, M., 1999, Physics Reports, 310, 97 doi:10.1016/S0370-1573(98)00080-5

[3] Birzan, L. et al. 2004, ApJ, 607, 800 doi:10.1086/383519

[4] Blundell, K.M., et al. 2006, ApJ, 644, L13 doi:10.1086/504839

[5] Challinor, A. \& Lasenby, A. 1998, ApJ, 499, 1 doi:10.1086/305623

[6] Colafrancesco, S., Marchegiani, P. \& Palladino, E. 2003, A\&A, 397, 27

[7] Colafrancesco, S. 2005, A\&A, 435, L9

[8] Colafrancesco, S., Profumo, S. \& Ullio, P.2006, A\&A, 455, 21

[9] Colafrancesco, S., Profumo, S. \& Ullio, P. 2007, PhRvD, 75, 3513

[10] Colafrancesco, S., 2007, New Astronomy Reviews, 51, 394 doi:10.1016/j . newar. 2006.11.067

[11] Colafrancesco, S. 2008, MNRAS, 385, 2041 doi:10.1111/j.1365-2966.2008.12961. $\mathrm{x}$ 
[12] Colafrancesco, S. 2010, Invited review at the Meeting "Multi-frequency behaviour of high-energy cosmic structures", MmSAIt, 81, 104

[13] Colafrancesco, S. 2010, Invited review at the Meeting "Astrophysics and cosmology after Gamow", 2010, AIPC, 1206, 5

[14] Colafrancesco, S. 2013, AcPol, 53, 560

[15] Colafrancesco, S. 2014, AAS, \#223 \# 34005

[16] Colafrancesco, S. \& Marchegiani, P., 2008, A\&A, 484,51

[17] Colafrancesco, S. \& Marchegiani, P., 2010, A\&A, 520,31

[18] Colafrancesco, S., Marchegiani, P. \& Buonanno, R. 2011, A\&A, 527, L1

[19] Colafrancesco, S. \& Marchegiani, P., 2011, A\&A, 535,108

[20] Colafrancesco, S. \& Marchegiani, P., 2014, A\&A, $562, \mathrm{~L} 2$

[21] Colafrancesco, S. et al., 2013, A\&A, 550, 92

[22] Colafrancesco, S., Tullio, M. and Emritte, S. 2014, preprint

[23] Colafrancesco, S., et al. 2014, A\&A in press, (2013arXiv1312.1846C)

[24] Croston, J.H. et al. 2005, ApJ, 626, 733 doi:10.1086/430170

[25] De Bernardis, P., Colafrancesco, S. et al. 2012, A\&A, 538, 86

[26] Dogiel, V., Colafrancesco, S., Ko, C.M. et al. 2007, A\&A, 461, 433

[27] Govoni, F. and Feretti, L. 2004, IJMPD, 13, 1549 doi:10.1142/S0218271804005080

[28] Harris, D.E. \& Krawczynski, H. 2002, ApJ, 565, 244 doi:10.1086/324544

[29] Itoh, N., Kohyama, Y. \& Nozawa, S. 1998, ApJ, 502, 7 doi:10.1086/305876

[30] Kataoka, J. et al. 2003, A\&A, 410, 833

[31] Kompaneets, A.S. 1957, Soviet Phys. JETP, 4, 730

[32] Lavaux, G. et al. 2004, MNRAS, 347, 729 doi:10.1111/j.1365-2966.2004.07265. x

[33] Maartes, R. 2011, arXiv:1104.1300

[34] McNamara, B. R. et al. 2000, AAS, 32.13211

[35] http://www.mpa-garching.mpg.de/galform/virgo /millennium/
[36] Million, E. T., \& Allen, S. 2009, MNRAS, 399, 1307 doi:10.1111/j.1365-2966.2009.15359.x

[37] Nozawa, S., Kohyama, Y. \& Itoh, N. 2010a, arXiv:1009.2311

[38] Nozawa, S., Kohyama, Y. \& Itoh, N. 2010b, PhRvD, 82, 3009

[39] Pfrommer, C., Ensslin, T., \& Sarazin, C. L. 2005, A\&A, 430, 799

[40] Phillips, P.R. 1995, ApJ, 455, 419 doi:10.1086/176589

[41] Planck Collaboration, 2011, A\&A 536, A8

[42] Planck Collaboration 2011, A\&A 536, A9

[43] Planck Collaboration 2011, A\&A 536, A10

[44] Planck Collaboration 2011, A\&A 536, A911

[45] Planck Collaboration 2011, A\&A 536, A12

[46] Planck Collaboration 2011, A\&A 536, A911

[47] Planck Collaboration 2011, arXiv:1112.5595P

[48] Prokhorov, D., Colafrancesco, S. et al. 2011a, A\&A, 529, 39

[49] Prokhorov, D., Colafrancesco, S. et al. 2011b, MNRAS, 416, 302

[50] Prokhorov, D. and Colafrancesco, S., 2012, MNRAS, 424, L49 doi:10.1111/j.1745-3933.2012.01284.x

[51] Rephaeli, Y. \& Lahav, O. 1991, ApJ, 372, 21 doi:10.1086/169950

[52] Rowe, B. \& Silk, J. 2010, MNRAS in press, arXiv:1005.4234v2

[53] Ryu, D. et al. 2003, ApJ, 593, 599

[54] Sazonov, S. \& Sunyaev, R. 1999, MNRAS, 310, 765 doi:10.1046/j.1365-8711.1999.02981.x

[55] Sunyaev, R.A. \& Zel'dovich, Y.B.: Comments Astrophys. Space Phys. 1972, 4, 173

[56] Sunyaev, R. A. \& Zeldovich, Ia. B. 1980, ARA\&A, 18, 537 doi:10.1146/annurev.aa.18.090180.002541

[57] Wolfe, B. \& Melia, F. 2006, ApJ, 638, 125 doi:10.1086/498227

[58] Wolfe, B. \& Melia, F. 2008, ApJ, 687, 193 doi:10.1086/591723

[59] Yamada, M. et al. 2010, AJ, 139, 2494

[60] Zemcov, M. et al. 2010, A\&A, 518, L16 